\title{
Femtosecond Laser-Induced Formation of Gold-Rich Nanoalloys from the Aqueous Mixture of Gold-Silver Ions
}

\author{
Yuliati Herbani, Takahiro Nakamura, and Shunichi Sato \\ Institute of Multidisciplinary Research for Advanced Materials (IMRAM), Tohoku University, Katahira 2-1-1, Aoba-ku, \\ Sendai 980-8577, Japan \\ Correspondence should be addressed to Shunichi Sato, sato@tagen.tohoku.ac.jp
}

Received 27 July 2010; Revised 25 September 2010; Accepted 5 October 2010

Academic Editor: Edward Andrew Payzant

Copyright (c) 2010 Yuliati Herbani et al. This is an open access article distributed under the Creative Commons Attribution License, which permits unrestricted use, distribution, and reproduction in any medium, provided the original work is properly cited.

The synthesis of gold-silver (AuAg) nanoalloys of various compositions has been performed by direct irradiation of highly intense femtosecond laser pulse in the presence of polyvinylpyrrolidone (PVP). The mixture of Au and Ag ions of low concentration was simply introduced into a glass vial and subjected to femtosecond laser pulses for several minutes. The AuAg nanoalloys of 2-3 nm with reasonably narrow size distribution were formed, and the position of the surface plasmon resonance (SPR) increased monotonically with an increase in the gold molar fraction in the ion solutions. The high resolution transmission electron microscope (HRTEM) images exhibited the absence of core-shell structures, and the energy dispersive X-ray spectroscopy (EDX) analysis confirmed that the particles were Au-rich alloys even for the samples with large fraction of $\mathrm{Ag}^{+}$ions fed in the solution mixture. The formation mechanism of the alloy nanoparticles in the high intensity optical field was also discussed.

\section{Introduction}

Bimetallic alloy nanoparticles have attracted much attention over the past decades, and much effort has been devoted to their fabrication (including homogenous alloys, core-shell and mixed particles) due to their synergetic and unique catalytic, electronic, and optical properties as a function of the composition, which are different from those of the monometallic constituents. For instance, absorption peak of surface plasmon resonance (SPR) for AuAg alloy nanoparticles can be tuned systematically from 520 to $400 \mathrm{~nm}$ by changing the alloy composition whereas plasmonic absorption for spherical $\mathrm{Au}$ and $\mathrm{Ag}$ nanoparticles is generally restricted to be around 520 and $400 \mathrm{~nm}$, respectively. In addition to the optical properties, AuAg alloy nanoparticles also exhibit a better and synergistic performance in their catalytic activity for $\mathrm{CO}$ oxidation as compared to that of either $\mathrm{Au}$ or $\mathrm{Ag}$ nanoparticles [1, 2]. Efforts for the fabrication of AuAg bimetallic nanoparticles have been also conducted by various techniques ranging from chemical methods using various reagents [3-5], to physical approaches using various energies such as $\gamma$-ray [6], microwave [7], and laser [8]. These attempts were done due to the understanding that the preparation conditions of the nanoparticle have a direct effect on the size, structure, and composition and consequently, on the resulting catalytical, electronic, and optical properties. Particularly for the structure, synthesis method determined whether the resulting bimetallic particles may exhibit alloy behavior [7], core-shell [8] system, or any other phase segregation behavior.

In the last few years, radiation-assisted processes have been used to obtain metal colloids, including the techniques using UV-visible light [9], laser [10], $\gamma$-rays [11], and Xray [12]. The techniques indeed offered several advantages such as no need of using the reducing agent and their versatility of producing small particles, but they were time consuming. For example, at least 5 hours for $\gamma$-rays, 30 hours for X-ray conventional sources, and even longer time for ultraviolet exposures were required to produce significant amounts of particles from a $3 \mathrm{ml}$ precursor solution. Of all the methodologies developed, direct irradiation of metal salts using femtosecond laser pulse is a novel technique for metal nanoparticles preparation and has not been extensively reported, especially for multimetallic system. Our method specifically uses highly intense femtosecond laser pulses to generate the optical breakdown of the containing medium 
following the generation of the free radicals responsible for the reduction process of metal ions. It only takes about 30 minutes for a complete reduction of 3-ml solution containing a midconcentration of metallic ions. In our previous works, the monometallic gold, platinum, and silver nanoparticles [13-15] have been successfully produced by direct laser irradiation of the corresponding metal salt solution containing no reducing agent using tightly focused femtosecond laser pulses. Due to the ultrashort laser-matter interaction time and the high peak power, the approach allows the formation of noble metal nanoparticles as small as $2 \mathrm{~nm}$ with a coefficient of variation below $30 \%$ in the presence of dispersant. These results were quite distinctive with those preformed by the former researches [16-18] with the same method but a weaker focusing condition and less peak power were used, resulting in a broader size distribution of metal nanoparticles. Succeeding the fabrication of monometallic nanoparticles with narrow size distribution, we proceed to use femtosecond laser pulse irradiation of metal salt solution as a novel technique that produces homogeneous alloy nanoparticles.

Hence, as a test case for bimetallic nanoparticle synthesis, in this study we use silver and gold because of their convenient optical properties to distinguish core-shell growth from alloy growth. Using femtosecond laser pulses with narrow pulse width and high peak power, AuAg alloy nanoparticles with size approaching 2-3 nm which are most preferable for catalytic purposes were formed in aqueous phase. The mechanism for the formation of bimetallic AuAg alloy nanoparticles in the high intensity optical field was also discussed.

\section{Experimental}

Colloidal dispersion of Au-Ag alloy nanoparticles was synthesized by direct irradiation of a mixed solution of gold and silver salts using highly intense femtosecond laser pulses at room temperature. All chemicals were used without further purification. Firstly, the solutions of gold and silver ions with the concentration of $1.32 \times 10^{-5} \mathrm{M}$ were prepared separately by dissolving $\mathrm{HAuCl}_{4} \cdot 3 \mathrm{H}_{2} \mathrm{O}$ (Sigma-Aldrich, +99.999\%) and $\mathrm{AgNO}_{3}$ (Sigma-Aldrich, +99.998\%) in extrapure water containing $0.01 \mathrm{wt} \%$ of polyvinylpyrrolidone (PVP) as a dispersant. The concentrations were fixed low to ensure the reaction quotient $(Q)$ to be $1.74 \times 10^{-10}$, safely below the $K_{\mathrm{sp}}$ of $\mathrm{AgCl}(\mathrm{s}) 1.8 \times 10^{-10}$ and hence, ensuring the complete solubility of $\mathrm{Ag}^{+}$in the presence of $\mathrm{Cl}^{-}$in the gold salt. The fresh aqueous solutions containing gold or silver ions were then mixed in appropriate volume to obtain solutions with $\mathrm{Au} / \mathrm{Ag}$ molar ratios of 90/10, 75/25, 50/50, $25 / 50$, and $10 / 90$ in a total volume of $3.0 \mathrm{ml}$. No turbidity caused by $\mathrm{AgCl}$ colloids formation was observed after the mixing. In laser irradiation experiment, the mixed solution was introduced into a rectangular quartz glass cuvette and irradiated for 9 minutes by the highly intense femtosecond laser pulses at the wavelength of $800 \mathrm{~nm}$ generated by a chirped pulse amplification system (Spitfire, Spectra-physics Co.). The typical pulse width, repetition rate, and maximum

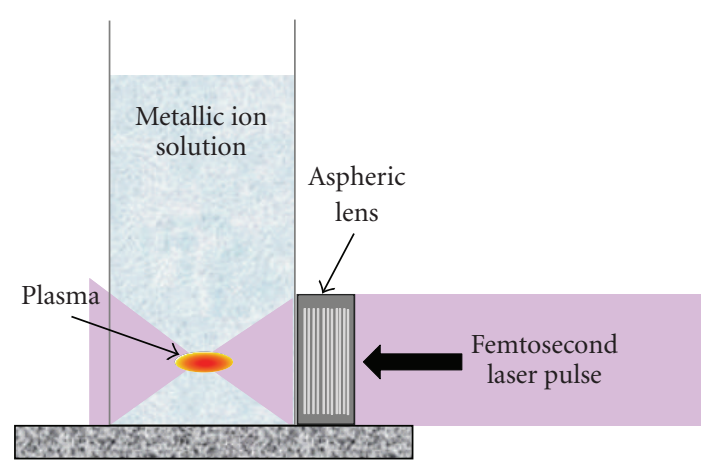

FIGURE 1: Set-up of irradiation experiment.

pulse energy per pulse were $100 \mathrm{fs}, 100 \mathrm{~Hz}$, and $6 \mathrm{~mJ}$, respectively. The laser beam was tightly focused using an aspheric lens with a focusing length of $8 \mathrm{~mm}(\mathrm{NA}=0.5)$ and directed perpendicularly to the surface of the cuvette into the solutions as shown in Figure 1. Considering the diameter of the laser beam before focusing was approximately $3.2 \mathrm{~mm}$, the theoretical laser intensity is about $3.2 \times 10^{18} \mathrm{~W} / \mathrm{cm}^{2}$ at the focal spot taking no account for the aberration. This intensity is expected to exceed the optical breakdown threshold of any solvent.

After irradiation, each sample was characterized by a UVvisible spectrometer (V630iRM, JASCO Co. Tokyo, Japan) to observe an SPR spectrum in $200-700 \mathrm{~nm}$ range and then confirm the nanoparticles formation. TEM experiments were performed using a JEOL2000-EXII (200 kV, JEOL Ltd., Tokyo) to obtain the electron micrographs for all samples. We also used a high-resolution transmission electron microscope (HRTEM, TITAN, FEI Co. London, UK) operating at $300 \mathrm{kV}$ accelerating voltage along with energy dispersive X-ray spectroscopy (EDX) analysis to feature the morphological structure and the composition of the particles fabricated in the solutions with the $\mathrm{Au} / \mathrm{Ag}$ concentration of $25 / 75,50 / 50$, and 75/25. For the TEM observations, several drops of sample were placed on a carbon-coated microgrid and left to dry in room temperature. The bright field image of each sample was then processed using image analysis software to measure the size of each nanoparticle. At least 500 particles were counted for each sample, taken from three separate areas on the microgrid.

\section{Results}

AuAg alloy nanoparticles were obtained through the simultaneous reduction of gold and silver ions at low concentrations using tightly focused femtosecond laser pulse in aqueous phase. Figure 2 shows UV-visible absorption spectra of the bimetallic colloids prepared with different Au/Ag molar ratio. These spectra were obtained shortly after irradiation synthesis. In general, there are several aspects that can be deduced from this figure. First, only a single plasmon absorption peak was observed for each sample, whose position shifts continuously from that of pure Au nanoparticles $(\sim 508 \mathrm{~nm})$ to that of pure Ag nanoparticles as a function of composition. 


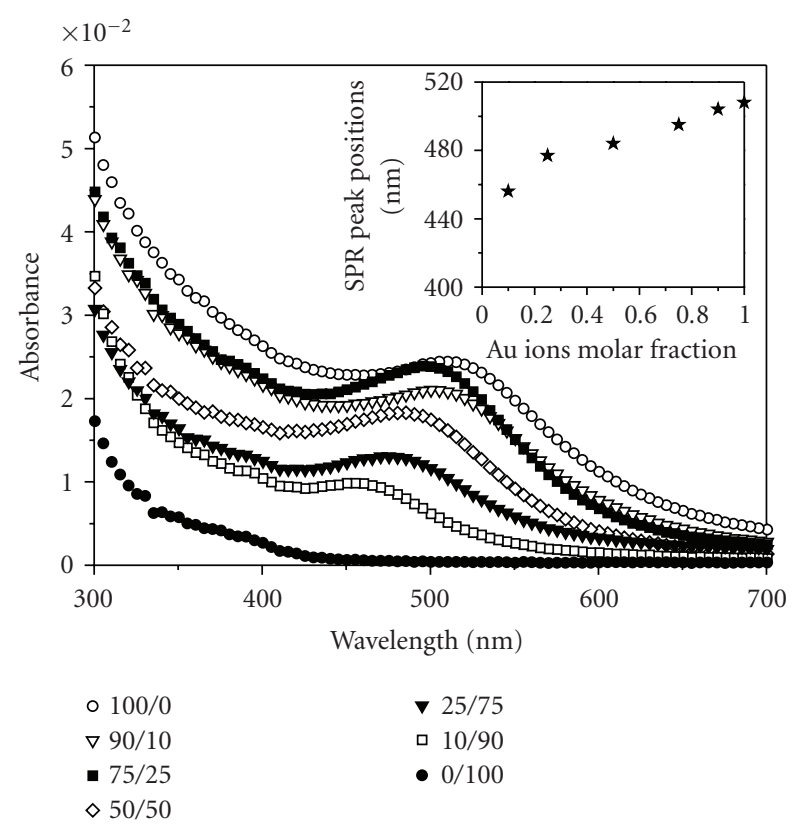

FIGURE 2: UV-Vis absorption spectra of aqueous solutions of AuAg nanoparticles with $\mathrm{Au} / \mathrm{Ag}$ molar ratio of 100/0, 90/10, 75/25, 50/50, $25 / 75,10 / 90$, and $0 / 100$ at $1.32 \times 10^{-5} \mathrm{M}$ just after irradiation using femtosecond laser pulses. Inset: the positions of the SPR are plotted against Au molar fraction in the reaction mixtures.

This result indicates that homogenous alloy nanoparticles were formed because single plasmon absorption peaks are obtained by neither the simple physical mixture of monometallic $\mathrm{Au}$ and $\mathrm{Ag}$ colloidal dispersion, nor the formation of core-shell $\mathrm{Au} / \mathrm{Ag}$ nanoparticles that have two absorption peaks as suggested by Mie's theory [19]. Second, it was also observed that the absorbances of all samples are weak and noisy due to low concentration of nanoparticle produced in the solution. Interestingly, surface plasmon intensity gradually decreases with an increase in Ag contents, indicating that the nanoparticle concentration seems to vary with initial feed ratio of metallic ions in the mixed solution. However, it is quite difficult to quantify the concentration of nanoparticles produced in the solution solely from UVvisible spectra since it involves a relatively long calculation route which requires precision data of particle size and extinction efficiency [20]. Third, it was also found that no SPR peaks of Ag nanoparticles were observed in the UVVis spectra indicates that Ag nanoparticles might be not produced at low concentration of Ag ion precursor. This result is in agreement with the previous study in our group [15] where monometallic Ag can only be formed at a higher concentration of about $0.3 \mathrm{M}$ under the same experimental condition. Finally, the surface plasmon absorptions for mixed solutions were located between those for pure gold and silver nanoparticles. As shown in the inset of Figure 2, the positions of the SPR are plotted against Au molar fraction in the reaction mixtures. In this inset, a monotonic variation of SPR peak position with Au molar fraction is observed (note that no SPR data for pure Ag sample was plotted on the graph because the absorption is very weak). Thus, it is implied that the concentration of $\mathrm{Au}$ and $\mathrm{Ag}$ ions in the initial mixture correlates to the alloy compositions, which will be confirmed later by EDX analysis. Furthermore, the peak position shifted to the longer wavelength region as the $\mathrm{Au} / \mathrm{Ag}$ molar ratio changed from $10 / 90$ to $90 / 10$. This finding also suggested that bimetallic nanoparticles formed were homogeneous alloys and thus, the specific absorption bands correspond to the SPR bands of AuAg alloy nanoparticles of a specific composition. Due to a low concentration, however, the color of the AuAg nanoparticle solutions observed by the naked eye was very faint as confirmed by a weak absorption intensity for each composition (Figure 2).

In order to determine the size and size distribution of the nanoparticles, TEM analysis has been performed. Figure 3 shows a representative set of TEM images for AuAg nanoparticles prepared in this work, and their respective size distributions are also presented. For pure Ag sample, it was quite hard to find Ag nanoparticles over the microgrid, so the size distribution was not shown here. On the other hand, for $\mathrm{Au}$ and $\mathrm{AuAg}$ nanoparticles, the individual nanoparticles appear to be well isolated. The size distribution analysis shows that the mean particle sizes are $2.22 \pm 0.6 \mathrm{~nm}$ and $2.97 \pm 1.9 \mathrm{~nm}$ for $\mathrm{Au} / \mathrm{Ag}=10 / 90$ and pure Au nanoparticles, respectively. Moreover, the particle sizes show a tendency to decrease as the Ag content increases although the mean particle size differs slightly for nanoparticles with different initial feed ratio of metal ions. The tendency might correlate with the different formation rate of $\mathrm{Au}$ and $\mathrm{Ag}$ atoms in the given solution. For the solution with low content of $\mathrm{Au}$ ions, it appears that small number of $\mathrm{Ag}$ ions were reduced to $\mathrm{Ag}$ atoms and then alloyed with $\mathrm{Au}$ atoms which are also available in a small amount according to its feeding ratio and hence resulted in the formation of alloy nanoparticle with a small particle size. Thus, it suggests that the presence of gold in the mixed solution facilitates the formation of silver atoms and hence lead to the formation of AuAg alloy nanoparticles.

Further support for the homogenously alloy structure of the AuAg nanoparticles has been provided by HRTEM images. Figure 4 shows the typical HRTEM micrograph of the nanoparticles prepared for initial Au/Ag ratios of 25/75, $50 / 50$, and 75/25. No core-shell structure was observed. The nanoparticles contrast appeared homogenous, and the presence of fringed facet implies that synthesized nanoparticles were highly crystalline. The fast Fourier transform (FFT) images are also presented in the insets in Figure 4. A brief morphological study of the small particles in our AuAg colloidal dispersion through the analysis of FFT images revealed that there is no trend of the class of the structures in each sample. Most of particles appeared to have undefined structures (Figure 4(c)) rather than simple face-centeredcubic (Figure 4(a)) or twinned structures (Figure 4(b)). We believe that particle size and morphology do not merely depend on the composition of elemental component, but mainly on the local condition of clustering process for individual particles.

To verify the composition of the nanoparticles, energy dispersive X-ray spectroscopy (EDX) analysis of the single isolated particles was carried out (Figure 5). Since there 

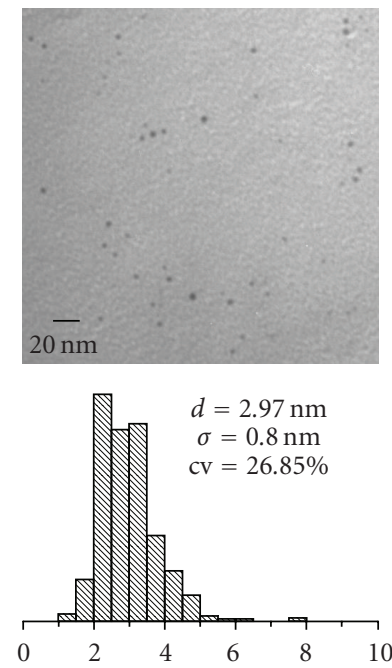

(a)
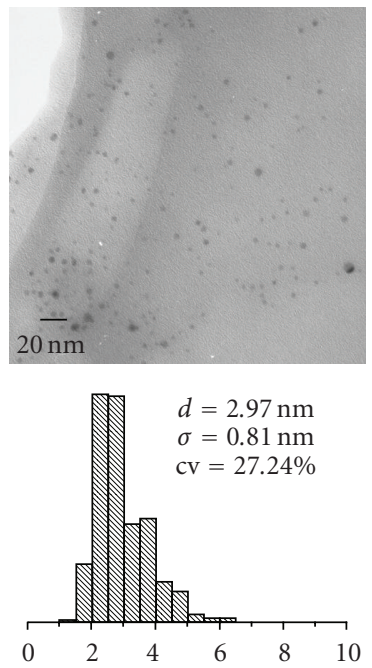

(b)
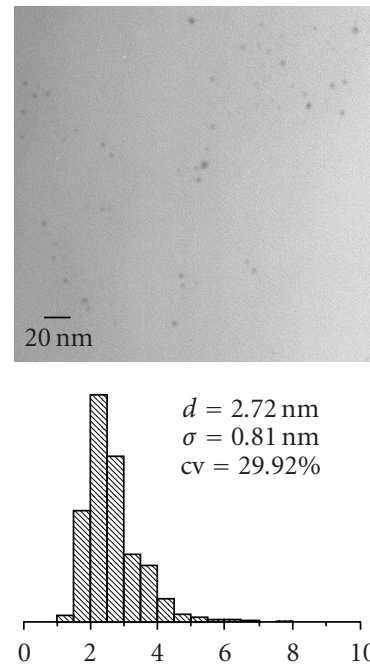

(c)
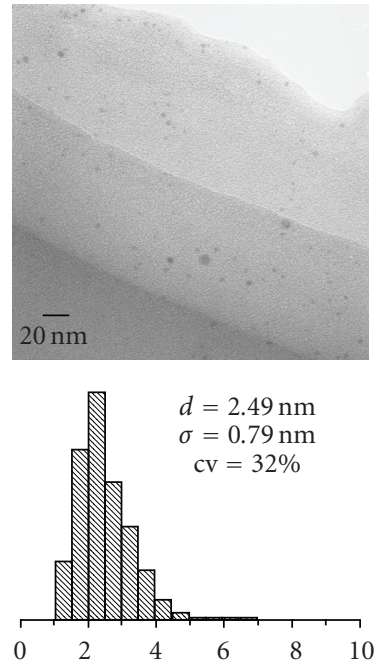

(d)
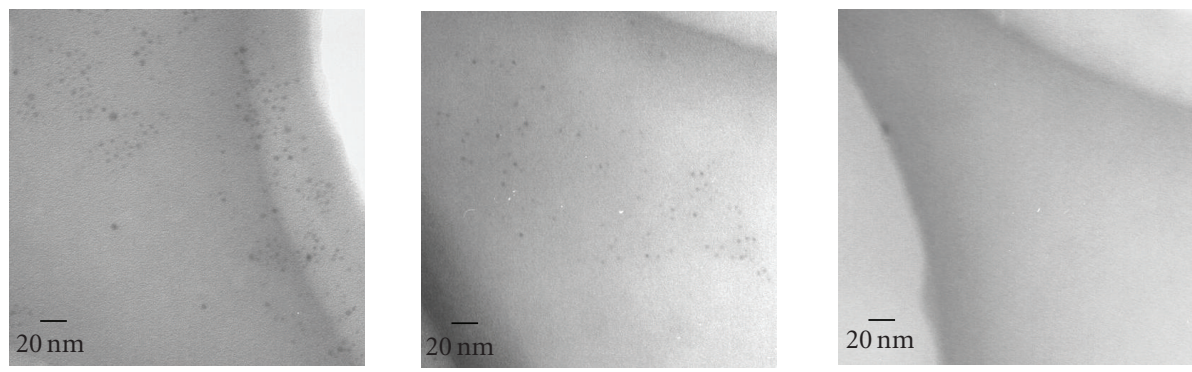

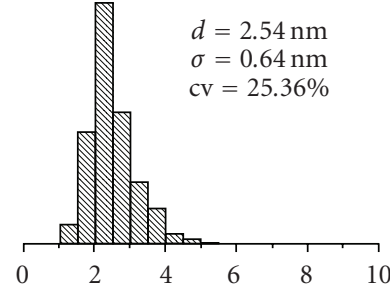

(e)

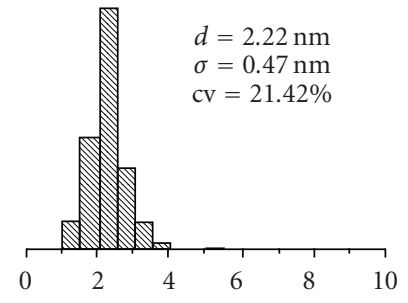

(f) (g)

FIGURE 3: TEM micrograph of samples prepared in this work: Au/Ag = (a) 100/0, (b) 90/10, (c) 75/25, (d) 50/50, (e) 25/75, (f) 10/90, and (g) $0 / 100$. The corresponding particle size distributions were also presented, except for (g) because no particles were found.

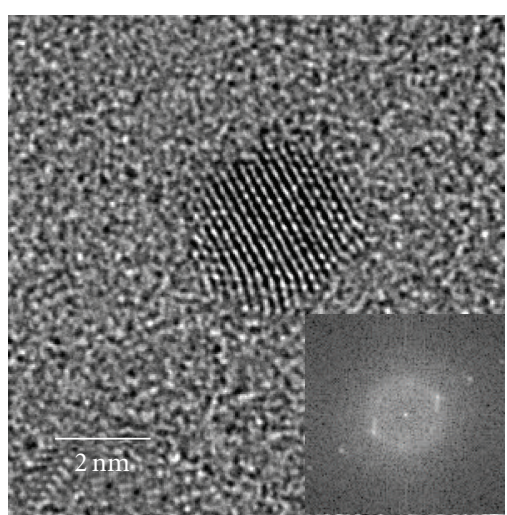

(a)

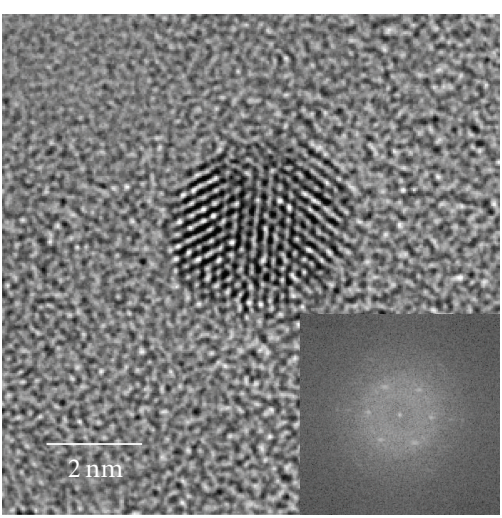

(b)

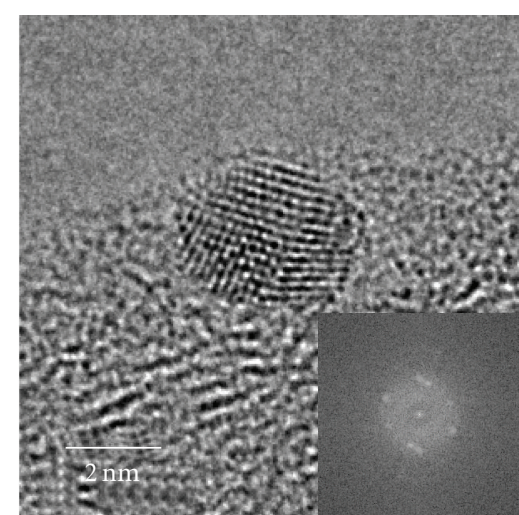

(c)

Figure 4: High resolution electron micrographs of typical bimetallic AuAg nanoparticles with initial concentration of (a) 25/75, (b) 50/50, and (c) $75 / 25$. Note that fringes pattern confirm the crystalline nature of nanoparticles. (Inset figure: the corresponding FFT images.) 
TABLE 1: EDX analysis for the fraction of the elemental Au in the nanoparticles with initial feeding ratio $\mathrm{Au} / \mathrm{Ag}=25 / 75,50 / 50$, and $75 / 25$.

\begin{tabular}{lcc}
\hline $\begin{array}{l}\text { The fraction of Au ions } \\
\text { in the mixed solution } \\
(\mathrm{mol} \%)\end{array}$ & \multicolumn{2}{c}{ EDX analysis } \\
\hline 25 & $67.20 \pm 13.45$ & $78.90 \pm 13.45$ \\
50 & $73.97 \pm 9.63$ & $83.49 \pm 9.63$ \\
75 & $87.69 \pm 10.02$ & $87.69 \pm 10.02$ \\
\hline
\end{tabular}

were only a few number of nanoparticles found in the microgrid, about 3 particles were chosen to analyze their average composition. It is found that the composition of individual particles varied from particle to particle for a given sample, and thus the average composition was used for discussion. According to the EDX analysis (Table 1), the $\mathrm{Au} / \mathrm{Ag}$ elemental ratios of bimetallic nanoparticles were determined to be $67.2 / 32.8,74 / 26$, and $87.7 / 12.3$ for samples with initial composition mixture of $\mathrm{Au} / \mathrm{Ag}$ ions $=25 / 75$, $50 / 50$, and $75 / 25$, respectively. This result suggested that the final compositions in the alloy particles were not the same as the initial feeding ratio in the solution mixtures even though the samples showed bimetallic nature and consists of two metal elements. Also, it is indeed intriguing that all samples predominantly contained Au atoms (Au-rich alloy particles) even for the samples with large concentration of Ag ions in the initial mixture. Assuming that Au ions were all consumed by the laser during the given irradiation time, only about $16.3 \%$ of $\mathrm{Ag}$ ions were converted to $\mathrm{Ag}$ atoms and alloyed with $\mathrm{Au}$ atoms in the resulted nanoparticles $(\mathrm{Au} / \mathrm{Ag}=67.2 / 32.8)$ for the sample with the initial ratio of $\mathrm{Au} / \mathrm{Ag}=25 / 27$. In similar way, in the samples with initial ratio of $50 / 50$ and $75 / 25$, the percentages of $\mathrm{Ag}$ ions that have been converted into $\mathrm{Ag}$ atoms were about $35 \%$ and $42 \%$, respectively. The percentage of the converted Ag ions increases with an increase of Au ions in the mixture solution. Thus, it seems that the presence of Au ions in the reaction mixture of low concentration advances the formation of $\mathrm{Ag}$ atoms in the alloy, clarifying the previous consideration deducted from the UV-visible spectra analysis of the samples (Figure 2).

\section{Discussions}

Metal nanoparticles are commonly synthesized by direct chemical reduction of metal ions using a reducing agent in solution due to the relative difference in the reduction potential between reducing agent and metal ions. In the present paper, metal atoms are produced by femtosecond laser pulse irradiation-induced reduction of metal ion precursors. Theoretically, the light intensity of the tightly focused laser beam used in this study is in the order of $10^{18} \mathrm{~W} / \mathrm{cm}^{2}$, which is high enough for the atoms and molecules in a condensed matter to absorb several photons simultaneously (multiphoton ionization) and induces the bound-free transition thus releasing free electrons. The process would

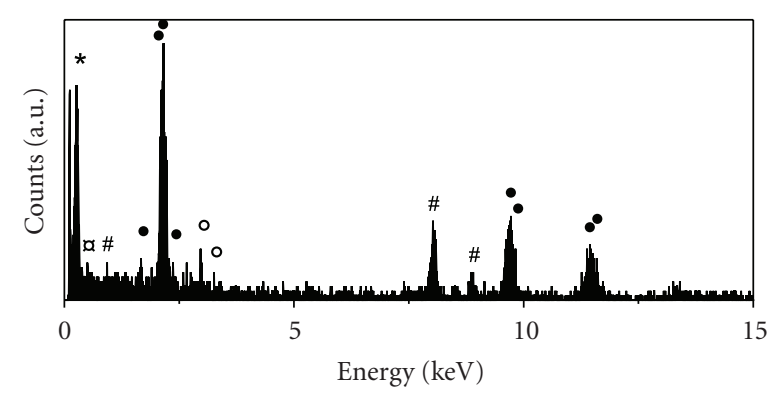

(a)

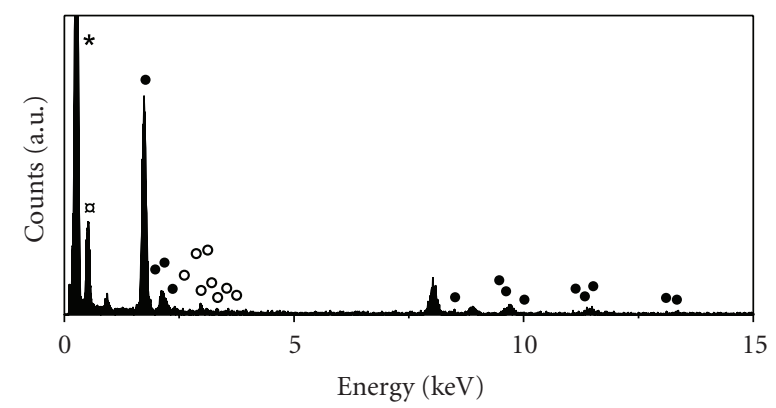

(b)

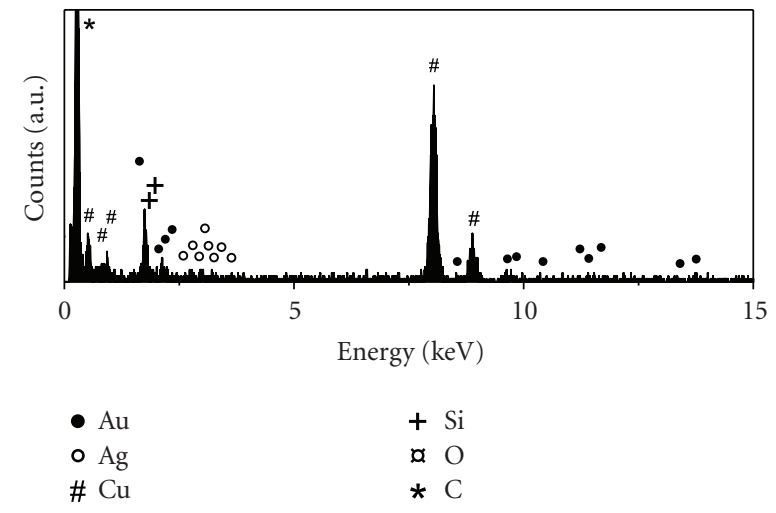

(c)

FIGURE 5: EDX pattern of bimetallic AuAg nanoparticles with initial concentration of (a) 25/75, (b) 50/50, and (c) 75/25.

easily provide a few free electrons with some initial kinetic energy created from molecules with low ionization potential in the focal volume at the front part of the pulse. The energetic electrons were accelerated further in the strong laser field and collided with atoms or molecules in the solution resulting in the ejection of the other few numbers of free electrons (collisional or avalanche ionization) and then inducing an optical breakdown of the bulk solution indicated by the presence of plasma and bubbles. Specifically, when an intense femtosecond laser field is focused in aqueous media containing metal ions, in which water molecules represent the most abundant species in the solution, the free electrons were provided by the dissociation of water molecules, besides the formation of the energetic radicals such as $\mathrm{H}^{\bullet}$ and $\mathrm{OH}^{\bullet}$ occurred $[21,22]$. These free electrons and hydrogen radicals contained in the plasma might be then caught by $\mathrm{H}^{+}$or $\mathrm{OH}^{-}$ions to form the bubbles of $\mathrm{H}_{2}$ and 


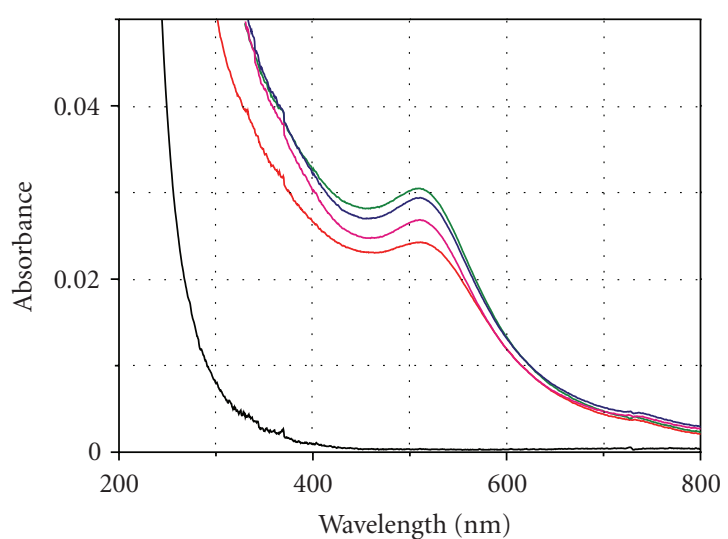

(a) $\mathrm{Au} / \mathrm{Ag}=100 / 0$

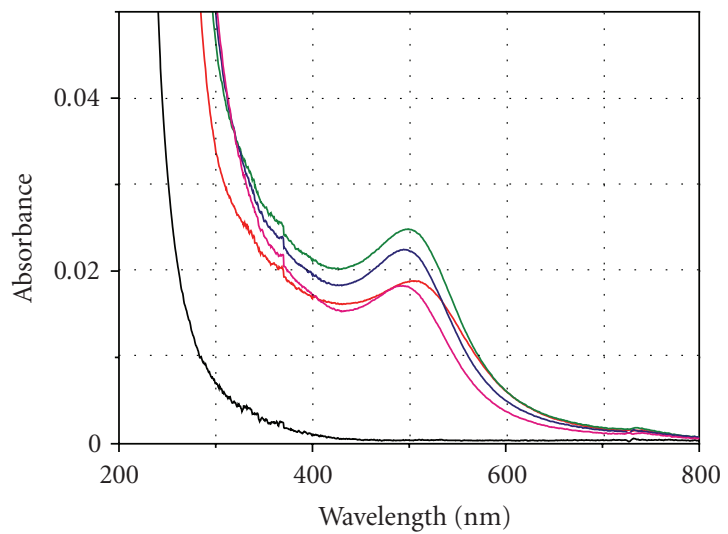

(c) $\mathrm{Au} / \mathrm{Ag}=75 / 25$

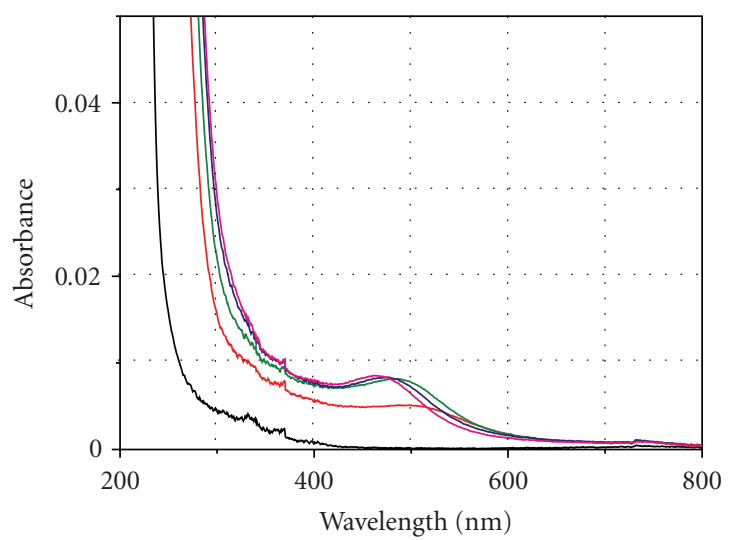

Irradiation time (min)

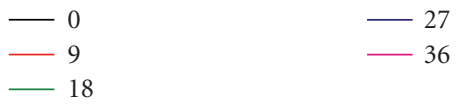

(e) $\mathrm{Au} / \mathrm{Ag}=25 / 75$

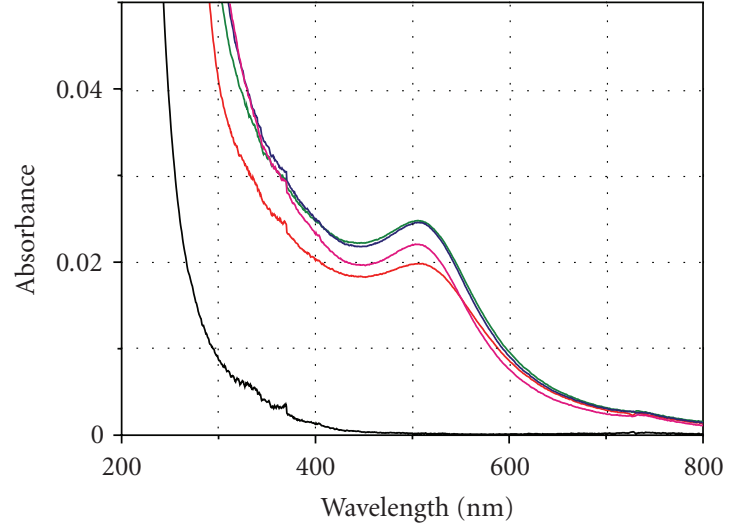

(b) $\mathrm{Au} / \mathrm{Ag}=90 / 10$

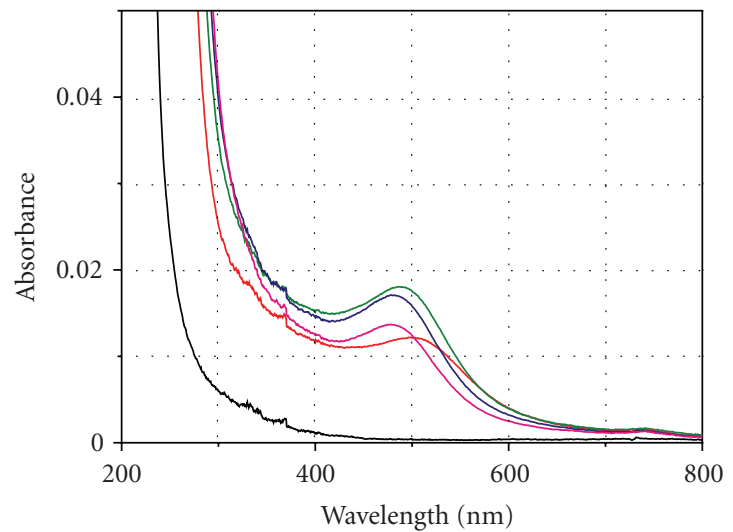

(d) $\mathrm{Au} / \mathrm{Ag}=50 / 50$

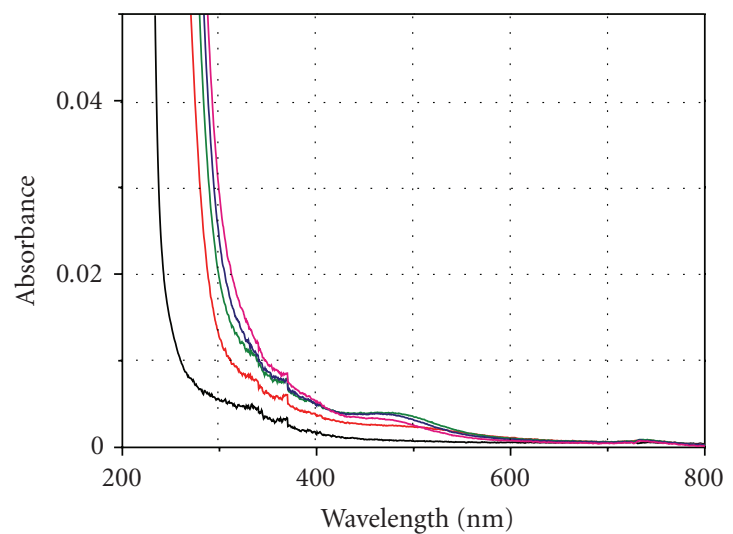

Irradiation time ( $\mathrm{min})$

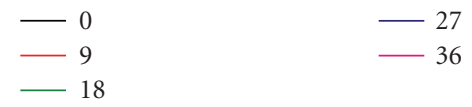

(f) $\mathrm{Au} / \mathrm{Ag}=10 / 90$

FIGURE 6: Evolution of the absorption spectra for various mixed solution of Au and Ag ions upon the course of femtosecond laser irradiation. The legend shows the irradiation time in minutes and is applied for all figures.

$\mathrm{O}_{2}$ gases as confirmed by gas chromatography test in the previous works $[16,17]$ and/or they can be trapped by metal ions, resulting in the formation of metal atoms during the femtosecond laser irradiation. It should be pointed out that plasma emission and tiny bubbles were also observed in this work. In contrast, $\mathrm{OH}^{\bullet}$ radicals are able to oxidize the ions or atoms into a higher oxidation state and thus counterbalance the reduction process governed by the free electrons and hydrogen radicals. However, because the mean free time of a free electron $(\sim 1 \mathrm{fs})$ released from an atom or molecules 
through multiphoton ionization in a solid or liquid has been known to be shorter than most of the femtosecond pulse durations used in the experiment [23], one may expect that there would be a few tens of cycles of collisional ionization for a whole $100 \mathrm{fs}$ pulse focused into a small focal zone resulting in optical breakdown, suppressing the oxidation rate caused by $\mathrm{OH}^{\circ}$, and thus allowing an efficient reduction process. Owing to the convection enhancement caused by plasma and bubbles, the laser energy can be delivered and deposited throughout the solution without stirring even though the irradiation point was on a fixed position in the solution. Whereas the formation mechanism for both monometallic gold $[13,16]$ and silver [24] from their corresponding metal salt using laser irradiation pulses has been proposed, now we are interested in revealing the alloying mechanism for bimetallic system.

In the case of bimetallic mixture of gold and silver, the solution to be irradiated contains the two metal precursors which are at low concentrations and thus stable in the presence of each other without $\mathrm{AgCl}$ precipitation. The strong reducing species (solvated electrons and free radicals) provided by femtosecond laser pulse readily react with both precursors. Even though the initial probabilities of being reduced by photolytic radicals and solvated electrons are the same for both metal precursors, the formation of gold atoms may prevail because reduction potential for $\mathrm{AuCl}_{4}{ }^{-}$ species $\left(E^{0} \mathrm{NHE}=+0.93 \mathrm{~V}\right)$ is greater than $\mathrm{Ag}^{+}$ions $\left(E^{0} \mathrm{NHE}=\right.$ $+0.799 \mathrm{~V})$. When $\mathrm{Au}$ ions are consumed by the reducing species, Ag ions are then reduced afterwards at the surface of Au. At this point, one would expect that core-shell structure is more likely to be formed than alloy structure. However, both the UV-Vis absorption spectroscopy in Figure 2 and HRTEM images have indicated the formation of AuAg alloy nanoparticles. It might indicate that high intensity delivered by femtosecond laser pulses may allow an efficient competition in favor of reduction at which alloying could be achieved in a very short time, shorter than any other chemical or electrochemical processes to be encountered, thus suppressing the possibility of metal segregation. Another possibility is that the core-shell structures might have been encountered at a certain stage during irradiation, and thus, the alloying process must be due to the spontaneous interdiffusion between the two atoms promptly after core-shell formation because of the similar atomic size of $\mathrm{Au}$ and $\mathrm{Ag}$ atoms. However, it is difficult to conclude the alloying mechanism of bimetallic nanoparticles only from their final absorption spectra or solely from the TEM images. To assess the alloying mechanism of the bimetallic nanoparticle produced in the intense femtosecond laser field, the evolution of the absorption spectra of mixed ion solutions during the irradiation course will be evaluated.

The additional experiment was conducted to reveal the alloying mechanism of $\mathrm{Au}$ and $\mathrm{Ag}$ atoms in the intense laser field by investigating the evolution of the absorption spectra of mixed ion solution upon the irradiation course. The experiment was done using the same irradiation condition, but the maximum pulse energy of laser is about $5.25 \mathrm{~mJ}$, a bit lower than that is used previously $(6 \mathrm{~mJ}$, see the experimental section). This was done based on the consideration that the complete reduction process by the reactive species is expected to take place in time longer than 9 minutes so that we can easily observe the evolution of the absorption spectra for various mixed solutions of $\mathrm{Au}$ and $\mathrm{Ag}$ ions. Figure 6 shows the absorption spectra for mixed solutions with gold molar fraction (GMF) ranging from 10 to $100 \%$ at irradiation times of $9,18,27$, and 36 minutes. As a consequence of lower laser energy used in the experiment, the result shows a quite different feature of absorption spectra at which the reduction process seems to be completed in longer than 9 minutes. At a 9-minute irradiation time (red line), the optical absorption spectra of various mixed solutions of $\mathrm{Au}$ and $\mathrm{Ag}$ ions present plasmon peak positions that are close to those of pure gold clusters (SPR position of pure Au is at $510 \mathrm{~nm}$; see Figure 6(a)), and then the peaks continue to increase and progressively shift to the blue region at 18minute irradiation. This result indicates that the gold atoms are formed first at the early stage, adsorb most of silver ions, and facilitate the reduction of Ag ions in the surface of $\mathrm{Au}$ atoms. Once $\mathrm{Ag}$ atoms were formed on the surface of $\mathrm{Au}$ atoms, interdifussion between the two atoms was pronounced, leading to the formation of homogenous $\mathrm{AuAg}$ alloy particles, as reported also in [18]. A small amount of $\mathrm{Ag}$ ions might be coreduced with $\mathrm{Au}$ ions at the early stage of irradiation but these reduced $\mathrm{Ag}$ atoms would then undergo a reverse oxidation through the electron transfer to the $\mathrm{Au}$ atoms because gold is more noble than silver in the presence of chloride ions [6]. That is why the peak positions of mixed ion solutions for 9-minute irradiation were very close to the SPR positions of pure Au nanoparticles. In other words, the so-called "chemical replacement reaction" in some extent occurred in the system. Additionally, the blue shift of the spectra at irradiation times longer than 9 minutes indicates that the nanoalloy composition also changed as the total number of silver atoms progressively increased and reached the compositional equilibrium in 18 minutes. However, the final compositions are still goldenriched from the view point of SPR positions. Moreover, when the irradiation was prolonged to 27 and 36 minutes for all samples, the absorption peaks decreased without changing its peak positions after reaching a maximum at 18minute irradiation. This might be induced by the oxidation due to the presence of oxygen that was accumulated in the excess irradiation of intense laser pulse even after the completion of nanoparticle production process.

Analyzing these results from the additional experiment, we now consider the peculiar behavior of the absorption spectrum for the sample with GMF of $75 \%$ in Figure 2. Although the SPR peak position shifted monotonically to the blue side with the decreasing in the gold molar fraction, the peak intensity only for the sample with the GMF of $75 \%$ increased. As shown in Figure 6, the SPR peak intensities for different gold molar fractions differ in their time evolution. Especially, the SPR peak for the GMF of $75 \%$ is dominant at 18-minute irradiation, indicating that the time needed to complete the particle formation depends on the GMF. Thus, this can also be attributed to the sudden increase of the SPR peak intensity of the spectra for the sample with GMF of 75\% in Figure 2. 
The formation mechanism of alloyed nanoparticle containing two metals of quite different redox properties in the intense laser field can be understood by considering the evolution of absorption spectra of the mixed solution. For the dilute mixed solutions of $\mathrm{Au}$ and $\mathrm{Ag}$ ions, the alloyed nanoparticles may be achieved at the certain stage of irradiation, and it is found that the final nanoparticles are gold-enriched, not segregated or core/shell ones, even though it was revealed that at the early stage $\mathrm{Au}$ atoms prevailed first rather than Ag atoms. As the irradiation time was prolonged, the alloying process into a homogenous nanoalloys may be facilitated by the interdifussion between the two atoms promptly after the reduction of less noble Ag atoms on the surface of more noble Au atoms due to the similar atomic size of $\mathrm{Au}$ and $\mathrm{Ag}$ atoms and the energetic environment provided by femtosecond laser irradiation. The formation of the alloy nanoparticles might also happen in the competition of oxidation that may exist due to the presence of oxygen and $\mathrm{OH}^{\bullet}$ in the solution. Nonetheless, the spectral evidence that showed the appearance of a single plasmon band in the visible range and the linear blue shift in the plasmon peak with the increase in Ag molar fraction (Figure 2) and also the lack of apparent core-shell structure in HRTEM images (Figure 3 ) fully supports the formation of homogenous gold-enriched alloy nanoparticle.

\section{Conclusion}

The AuAg alloy nanoparticles were successively prepared by femtosecond laser irradiation of the corresponding ions mixture at low concentration. Despite that the production yield is low, we verified the alloy formation from the fact that UV-visible absorption spectra continuously shifted with varying gold molar fraction and showed a single plasmon absorption band for all samples with different composition of metal ions in the solution. TEM images also exhibited the absence of core-shell structures. Furthermore, EDX analysis confirmed that the particles are Au-rich alloys even for the samples with large amount of $\mathrm{Ag}^{+}$ions fed in the solution mixture. The electron transfer from the coreduced Ag atoms to $\mathrm{Au}$ ions was also considered as a factor that causes the formation of this gold-enriched nanoalloy. The experimental results also revealed that monometallic Ag nanoparticles were hardly produced in this case, and their formation at low concentration requires either Au ions or atoms to be coexisted in the solution. This result is consistent with our previous work of silver synthesis using the same method. Mechanism of the alloy formation has also been proposed, and it was revealed that the gold atoms prevail first at the early stage before completely alloyed with Ag atoms at the certain stage of irradiation.

\section{Acknowledgments}

This paper was partially supported by the Research Subsidy Program for Young Researchers of financial year 2009 of GCOE "Material Integration International Centre of Education and Research," Tohoku University and also by the Japanese Science and Technology (JST) Agency.

\section{References}

[1] J.-H. Liu, A.-Q. Wang, Y.-S. Chi, H.-P. Lin, and C.-Y. Mou, "Synergistic effect in an Au-Ag alloy nanocatalyst: $\mathrm{CO}$ oxidation," Journal of Physical Chemistry B, vol. 109, no. 1, pp. 40-43, 2005.

[2] C.-W. Yen, M.-L. Lin, A. Wang, S.-A. Chen, J.-M. Chen, and C.-Y. Mou, "CO oxidation catalyzed by Au-Ag bimetallic nanoparticles supported in mesoporous silica," Journal of Physical Chemistry C, vol. 113, no. 41, pp. 17831-17839, 2009.

[3] D.-H. Chen and C.-J. Chen, "Formation and characterization of Au-Ag bimetallic nanoparticles in water-in-oil microemulsions," Journal of Materials Chemistry, vol. 12, no. 5, pp. 15571562, 2002.

[4] A. Pal, S. Shah, and S. Devi, "Preparation of silver, gold and silver-gold bimetallic nanoparticles in w/o microemulsion containing TritonX-100," Colloids and Surfaces A, vol. 302, no. 1-3, pp. 483-487, 2007.

[5] A. Pal, S. Shah, and S. Devi, "Preparation of silver-gold alloy nanoparticles at higher concentration using sodium dodecyl sulfate," Australian Journal of Chemistry, vol. 61, no. 1, pp. 66$71,2008$.

[6] M. Treguer, C. De Cointet, H. Remita et al., "Dose rate effects on radiolytic synthesis of gold-silver bimetallic clusters in solution," Journal of Physical Chemistry B, vol. 102, no. 22, pp. 4310-4321, 1998.

[7] P. Raveendran, J. Fu, and S. L. Wallen, "A simple and "green" method for the synthesis of $\mathrm{Au}, \mathrm{Ag}$, and $\mathrm{Au}-\mathrm{Ag}$ alloy nanoparticles," Green Chemistry, vol. 8, no. 1, pp. 34-38, 2006.

[8] G. Compagnini, E. Messina, O. Puglisi, R. S. Cataliotti, and V. Nicolosi, "Spectroscopic evidence of a core-shell structure in the earlier formation stages of Au-Ag nanoparticles by pulsed laser ablation in water," Chemical Physics Letters, vol. 457, no. 4-6, pp. 386-390, 2008.

[9] M. Sakamoto, M. Fujistuka, and T. Majima, "Light as a construction tool of metal nanoparticles: synthesis and mechanism," Journal of Photochemistry and Photobiology C, vol. 10, no. 1, pp. 33-56, 2009.

[10] S. Besner, A. V. Kabashin, F. M. Winnik, and M. Meunier, "Synthesis of size-tunable polymer-protected gold nanoparticles by femtosecond laser-based ablation and seed growth," Journal of Physical Chemistry C, vol. 113, no. 22, pp. 95269531, 2009.

[11] T. Li, H. G. Park, and S.-H. Choi, " $\gamma$-Irradiation-induced preparation of $\mathrm{Ag}$ and $\mathrm{Au}$ nanoparticles and their characterizations," Materials Chemistry and Physics, vol. 105, no. 2-3, pp. 325-330, 2007.

[12] C.-H. Wang, C.-J. Liu, C.-L. Wang et al., "Intense Xray induced formation of silver nanoparticles stabilized by biocompatible polymers," Applied Physics A, vol. 97, no. 2, pp. 295-300, 2009.

[13] T. Nakamura, Y. Mochidzuki, and S. Sato, "Fabrication of gold nanoparticles in intense optical field by femtosecond laser irradiation of aqueous solution," Journal of Materials Research, vol. 23, no. 4, pp. 968-974, 2008.

[14] T. Nakamura, K. Takasaki, A. Ito, and S. Sato, "Fabrication of platinum particles by intense, femtosecond laser pulse irradiation of aqueous solution," Applied Surface Science, vol. 255, no. 24, pp. 9630-9633, 2009.

[15] T. Nakamura, H. Magara, Y. Herbani, and S. Sato, "Fabrication of silver nanoparticles by high-intensity of laser irradiation of aqueous solution," in The proceeding of the 7th International Symposium on Atomic Level Characterization for New Materials 
and Devices (ALC '09), 10P10, Maui - Hawai, pp. 507-510, December 6-11, 2009.

[16] C. Zhao, S. Qu, J. Qiu, and C. Zhu, "Photoinduced formation of colloidal Au by a near-infrared femtosecond laser," Journal of Materials Research, vol. 18, no. 7, pp. 1710-1714, 2003.

[17] C. Zhao, S. Qu, J. Qiu, C. Zhu, and K. Hirao, "Preparation of colloidal Au by a femtosecond laser," Chemistry Letters, vol. 32, no. 7, pp. 602-603, 2003.

[18] H. Zeng, C. Zhao, J. Qiu, Y. Yang, and G. Chen, "Preparation and optical properties of silver nanoparticles induced by a femtosecond laser irradiation," Journal of Crystal Growth, vol. 300, no. 2, pp. 519-522, 2007.

[19] H. M. Chen, R. S. Liu, L.-Y. Jang, J.-F. Lee, and S. F. $\mathrm{Hu}$, "Characterization of core-shell type and alloy $\mathrm{Ag} / \mathrm{Au}$ bimetallic clusters by using extended X-ray absorption fine structure spectroscopy," Chemical Physics Letters, vol. 421, no. 1-3, pp. 118-123, 2006.

[20] W. Haiss, N. T. K. Thanh, J. Aveyard, and D. G. Fernig, "Determination of size and concentration of gold nanoparticles from UV-Vis spectra," Analytical Chemistry, vol. 79, no. 11, pp. 4215-4221, 2007.

[21] D. N. Nikogosyan and D. A. Angels, "Formation of free radicals in water under high-power laser UV irradiation," Chemical Physics, vol. 77, no. 1, pp. 208-210, 1980.

[22] S. L. Chin and S. Lagacé, "Generation of $\mathrm{H}_{2}, \mathrm{O}_{2}$, and $\mathrm{H}_{2} \mathrm{O}_{2}$ from water by the use of intense femtosecond laser pulses and the possibility of laser sterilization," Applied Optics, vol. 35, no. 6, pp. 907-911, 1996.

[23] L. Chin, Femtosecond Laser Filamentation, Springer, London, UK, 2009.

[24] J. P. Abid, A. W. Wark, P. F. Brevet, and H. H. Girault, "Preparation of silver nanoparticles in solution from a silver salt by laser irradiation," Chemical Communications, no. 7, pp. 792-793, 2002. 

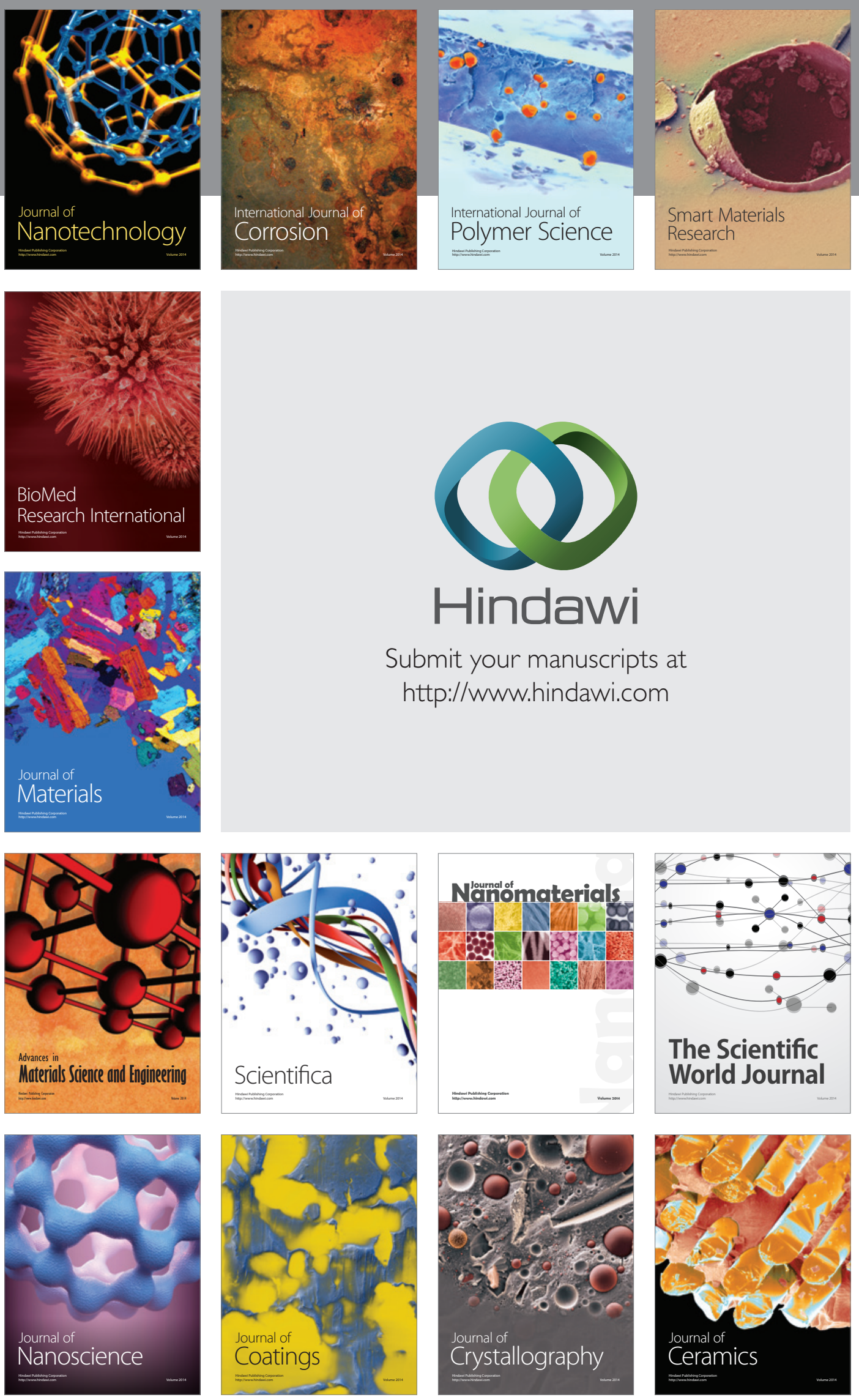

The Scientific World Journal

Submit your manuscripts at

http://www.hindawi.com

\section{World Journal}

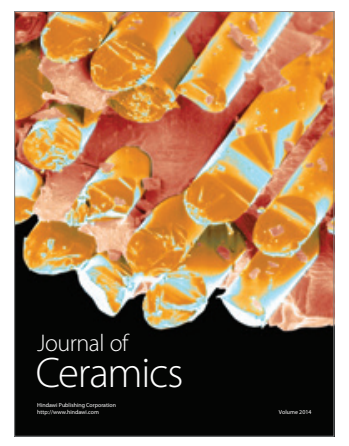

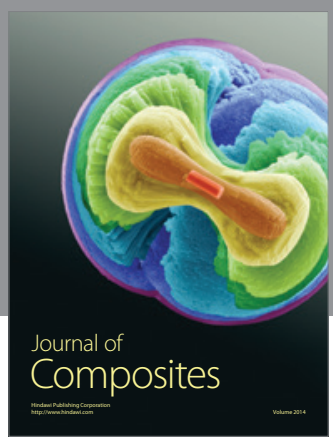
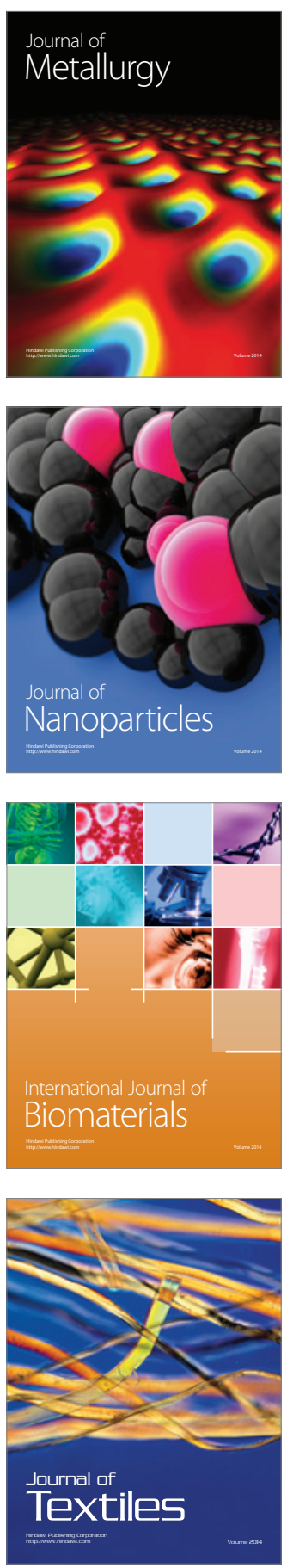